\title{
Spectral breaks for theorists
}

\section{Matthew G. Baring}

MUCH has been made over the past nine months of the spatial distribution of astronomical $\gamma$-ray bursts, as revealed ${ }^{1}$ by the BATSE (Burst and Transient Source Experiment) detector aboard the Compton Gamma-Ray Observatory satellite, in the hope this will tell us something of their origin. But more information may be forthcoming from the detailed spectra of individual bursts, recorded by the same apparatus. Schaefer et al. ${ }^{2}$ report finding distinct breaks at energies between $400 \mathrm{keV}$ and $2 \mathrm{MeV}$ in the spectra of five out of 18 bright bursts recorded by BATSE, which may be related to the source conditions.

The principal finding from BATSE is that the bursts are distributed isotropically over the sky, but that there is a comparative deficit of distant sources. The common inference is that the bursts are cosmological in origin $^{3}$, and cannot be explained in terms of the conventional hypothesis $^{4}$ involving neutron stars in our own (disk-like) Galaxy. The neutron-star hypothesis was most attractive because of the appearance of absorption features at less than $50 \mathrm{keV}$ in burst spectra recorded by the early KONUS experiments. These were naturally explained as cyclotron absorption lines in $10^{12}$-gauss magnetic fields expected of neutron stars. In 1988, the Ginga X-ray satellite observed sources with double absorption dips, equally spaced in energy ${ }^{5,6}$. This dramatically bolstered the cyclotron-line interpretation, and the idea that $\gamma$-ray bursts originate near neutron stars became the vogue until the BATSE detections of the past year.

\section{High quality}

The high quality of the BATSE burst data has made it possible now to discriminate between theoretical models of their spectra. The correct prediction of the shape of the energy spectrum near the break can now be made a test for any model, and should be a focus of future theoretical investigations. All reasonable source models for bursts require that an enormous luminosity is generated in a relatively small volume. Only a few radiation processes can accomplish this while also providing relatively sharp breaks in the $\gamma$-ray continuum.

Two-photon production of electronpositron pairs $\left(\gamma \gamma \rightarrow \mathrm{e}^{+} \mathrm{e}^{-}\right)$is the most familiar radiation mechanism for attenuating $\gamma$-ray spectra of astrophysical

sources. For a power-law spectrum of negative logarithmic slope (spectral index), the probability of attenuation increases with energy so that above some $\gamma$-ray threshold energy there is an absorption turnover and break in the spectrum. The higher the photon density in the source, which means a greater distance to the source for a given detected flux of radiation, the lower is the energy at which the break occurs. This property was used to place burst sources that showed no evidence of attenuation below about $3 \mathrm{MeV}$ (like many of the Solar Maximum Mission and BATSE

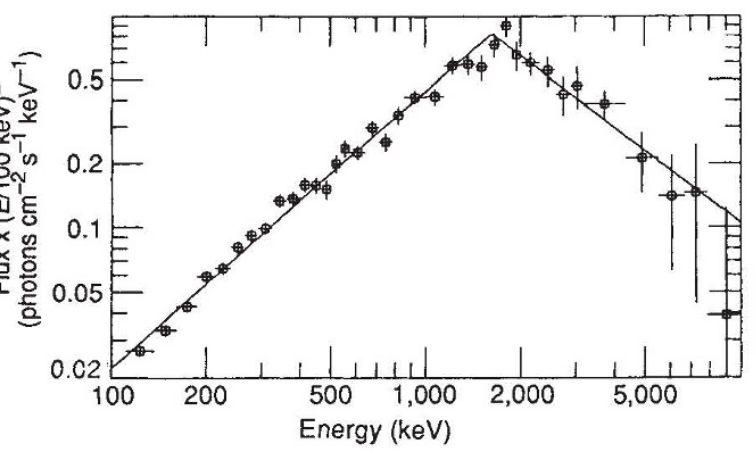

break yet: BATSE spectra reveal onset of $\gamma$ y-attenuating mechanisms. (Event 910814-69275, from ref. 2.)

theories of pulsar magnetospheres. Averaging over suitable radiation angular distributions, $\gamma \rightarrow \mathrm{e}^{+} \mathrm{e}^{-}$usually causes a sharp spectral break $^{8}$ between 1 and $3 \mathrm{MeV}$, compatible with two of the spectral breaks displayed by Schaefer $e t$ $a l^{2}$. But in such strong fields, synchrotron radiation is likely to be the dominant emission process, yielding relatively steep spectra so that only one of the BATSE examples could fit a model with $\gamma \rightarrow \mathrm{e}^{+} \mathrm{e}^{-}$attenuation.

\section{Relativistic electrons}

A third mechanism for generating spectral breaks is the inverse Compton scattering of X-ray photons (perhaps from an accretion disk or a stellar surface) by relativistic electrons, which give up some of their energy to the radiation. This could apply to bursts in both galactic and cosmological environments. In low magnetic fields, the continuum photons may be scattered many times to generate a peak around $1 \mathrm{MeV}$ of quasi-thermal shape ${ }^{9}$.

But nothing like this is seen. In the magnetic fields of neutron stars, inverse Compton scattering is a process that is resonant at the cyclotron energy (about 30 $\mathrm{keV}$ ) and can generate spectral breaks at any energy above this ${ }^{10}$. Despite its flexibility, this mechanism is quite constrained in its range of spectral slopes at

sources) within 1 kiloparsec of the Earth $^{7}$ (much closer, say, than the Galactic Centre). The breaks generated by $\gamma \gamma \rightarrow \mathrm{e}^{+} \mathrm{e}^{-}$are sharp enough to account for the results presented by Schaefer et al. ${ }^{2}$ but may not produce the appropriate slopes for the spectra above the breaks.

For extremely high source luminosities, as required for extragalactic bursts, the pairs produced will give a dense gas that will scatter the photons through the Compton process and produce a thermal peak in the spectrum, contrary to the data. To avoid this, the pair production rate must then be dramatically lower. This can be achieved if the $\gamma$-rays emerge in a beam more tightly collimated than known in extragalactic jets. The absence or presence of a spectral break should severely constrain the degree of radiation beaming in any model with a cosmological population of burst sources.

In the strong magnetic fields surrounding neutron stars, there is also the exotic quantum process of spontaneous production of an electron-position pair by a single photon, $\gamma \rightarrow \mathrm{e}^{+} \mathrm{e}^{-}$. This process has a threshold around $1 \mathrm{MeV}$, and will efficiently attenuate a $\gamma$-ray burst spectrum above this. It is a popular mechanism for the production of pairs in energies below the break, and is probably compatible with only two of the broken-power-law BATSE observations presented by Schaefer et al. ${ }^{2}$.

It is unlikely that the BATSE spectra will be surpassed this decade, and astrophysicists will be challenged to make the most of them. Certainly, no definitive answers are forthcoming yet. It is a pity that the most distant bursts will be too faint to yield spectra, as any redshift in their features relative to those in brighter bursts would confirm a cosmological origin. But fitting the energy of any break and the spectral slopes around it is still a powerful tool for unravelling the mechanism of $\gamma$-ray bursts.

Matthew G. Baring is in the Department of Physics, North Carolina State University, Raleigh, North Carolina 27695, USA.

1. Meegan, C. et al. Nature 355, 143-145 (1992).

2. Schaefer, B. E. et al. Astrophys. J. 393, L51-L54 (1992).

3. Mao. S. \& Paczynski, B. Astrophys. J. 388, L45-L48 (1992).

4. Mao, S. \& Paczynski, B. Astrophys. J. 389, L13-L16 (1992).

5. Fenimore, E. E. et al. Astrophys. J. 335, L71-L74 (1988).

6. Murakami, T. et al. Nature 335, 234-235 (1988)

7. Schmidt, W. K. H. Nature 271, 525-527 (1978)

8. Baring, M. G. Mon. Not. R. astr. Soc. 244, 49-57 (1990).

9. Zdziarski, A. A., Coppi, P. S. \& Lamb, D. Q. Astrophys. J. 357, 149-160 (1990).

10. Dermer, C. D. Astrophys. J. 347, L13-L16 (1989). 